\title{
Current trends in carbon nanotubes and their applications
}

\author{
Suresh Sagadevan $^{1}$, R. Varatharajan ${ }^{2}$ \\ ${ }^{1}$ Department of Physics, Sree Sastha Institute of Engineering and Technology, Chembarambakkam, Chennai, 600123, India \\ ${ }^{2}$ Department of Mechanical Engineering, Sree Sastha Institute of Engineering and Technology, Chembarambakkam, Chennai, India
}

\section{Email address:}

sureshsagadevan@yahoo.co.in(S. Sagadevan)

\section{To cite this article:}

Suresh Sagadevan, R. Varatharajan. Current Trends in Carbon Nanotubes and Their Applications. American Journal of Nanoscience and Nanotechnology. Vol. 1, No. 4, 2013, pp. 79-82. doi: 10.11648/j.nano.20130104.11

\begin{abstract}
Carbon based nanostructure materials exhibit unique mechanical, electrical, and optical characteristics, which may result in many unique device designs. These materials are biocompatible, chemically inert but capable of altering their electronic properties in the presence of some chemical species, and dimensionally compatible with biomolecules. They have interesting electronic characteristics, thus rendering them as potential chemical and biosensors. The recent progress in nanostructured materials and their possible applications in chemical and biological sensors could have a significant impact on data collection, processing, and recognition. This investigation is aimed towards evaluating the applications of nanostructures of carbon and giving a consolidated view of the structure, properties and applications of carbon nanotubes, with the aim of drawing attention to useful available information and to enhancing the interest in this new highly advanced technological field for the researcher and the manufacturing engineer.
\end{abstract}

Keywords: Nanotechnology, Nanostructured Materials, Carbon Nanotubes

\section{Introduction}

Nanotechnology and Nanoscience is the fastest developing field of science, which has found importance and intense interest for many researchers. The unique chemical, physical, electronic (metallic or semiconducting) and high thermal properties of carbon nanotubes (CNTs) have made them interesting materials for widespread application in fields, such as electrochemical sensors, biosensors, supports for heterogeneous metal catalysts in organic synthesis, fuel cells, semiconductors, batteries, random access memory cells, field effect transistor, field emission display, atomic force microscopy probes, microelectrodes, specific adsorbents to remove organic pollutants from water and waste water and as potential drug carriers in cancer therapy. The poor solubility of carbon nanotubes in organic solvents restricts them from being used as drug delivery agents into living systems in drug therapy. Hence, many modification approaches like physical, chemical or a combination of both have been exploited for their homogeneous dispersion in common solvents to improve their solubility [1-4]. The applications of carbon nanotubes (CNTs) range from quantum wire interconnects [5], diodes and transistors for computing [6], to high power electrochemical capacitors [7], data storage devices [8], field emitters for flat panel displays [9-11] and terahertz oscillators [12].
The reports that have appeared till now in the literature reveal that some modification is required to control the dispersion and such modification introduces specific functionalities as molecular wedges onto the surface of the carbon nanotubes. Thus, the functional groups present on the surface control the lateral interactions between the bundles and separate individual tubes. Hence, a homogeneous dispersion can be achieved by breaking the close lateral contact between them, which enhances the affinity towards solvents, and other related matrices [2]. Due to these specific properties, many research groups explored the development of novel methods for the modification of nanotubes and investigated the mechanistic aspects of these new classes of novel materials, called chemically modified carbon nanotubes (CMCNTs). A nanotechnology-based sensor platform will enable the direct detection of biological and chemical agents in a label-free, highly multiplexed format, over a broad dynamic range. This platform utilizes functionalized nanotubes, nanowires, and nanoparticles to detect molecular binding with high sensitivity and selectivity. The platform is capable of detecting a broad range of molecules, viz., DNA, RNA, proteins, ions and cells, and even the $\mathrm{pH}$ values. Detection is possible in both the liquid and gas phases, and is highly multiplexable, allowing for the parallel detection of multiple agents. The present paper reveals the structure, properties and applications of carbon nanotubes. 


\section{Introductions to Carbon Nanotubes}

Carbon nanotubes (CNTs; also known as buckytubes) are allotropes of carbon with a cylindrical nanostructure. Nanotubes have been constructed with length-to-diameter ratio of up to $132,000,000: 1$, which is significantly larger than that of any other material. These cylindrical carbon molecules have novel properties that make them potentially useful in many applications in nanotechnology, electronics, optics and other fields of materials science, as well as potential uses in architectural fields. They exhibit extraordinary strength and unique electrical properties, and are efficient thermal conductors. Nanotubes are members of the fullerene structural family, which also includes the spherical buckyballs. The ends of a nanotube might be capped with a hemisphere of the buckyball structure. Their name is derived from their size, since the diameter of a nanotube is in the order of a few nanometers (approximately 1/50,000th of the width of a human hair), while they can be up to 18 centimeters in length (as of 2010). Nanotubes are categorized as single-walled nanotubes (SWNTs) and multi-walled nanotubes (MWNTs).

\section{Structures of Carbon Nanotubes}

Carbon nanotubes occur in two general forms: SWCNTs and multi-walled carbon nanotubes MWCNTs. SWCNTs can generally be visualized as a single sheet of graphene that has been rolled into a hollow tubular shape. The orientation of the graphene sheet as it is rolled will dictate the resulting structure of the CNT. SWCNTs can have diameters as small as $0.4 \mathrm{~nm}$ and normally no larger than $2 \mathrm{~nm}$. MWCNTs can be viewed as several concentric SWCNTs with the outside diameters ranging between $5 \mathrm{~nm}$ and 100 $\mathrm{nm}$. The interlayer spacing of MWCNTs is approximately $0.34 \mathrm{~nm}$ and this value is also widely taken as the thickness of the individual CNT layers in numerical simulations and in some experimental investigations. Fig.1 depicts both the SWCNT and MWCNT structures.

\section{Carbon Nanotubes in Energy Storage}

Inducing purposeful defects in the structures of carbon nanotubes has given them the ability to perform as super capacitors; this has huge implications for the future of energy storage, batteries and electronic devices that rely on stored energy. The research in this area has already resulted in the development of energy storage devices, which are smaller, have higher storage capacity and can be recharged far quicker than the existing batteries, and can be a leap in terms of nanotechnology. Student research groups are reported to have developed paper-thin batteries made of cellulose embedded with carbon nanotubes. The thin paper-like structure which is also flexible could allow these to be stacked in larger amounts for greater energy requirements and can well redefine the way we see batteries and energy storage devices in our everyday devices. With con- siderable research going on around the use of carbon nanotubes to come up with solutions for energy storage, it can well be the innovation that shapes the advances in future material science and nanotechnology. Fig.2 shows the energy storage devices of carbon nanotubes

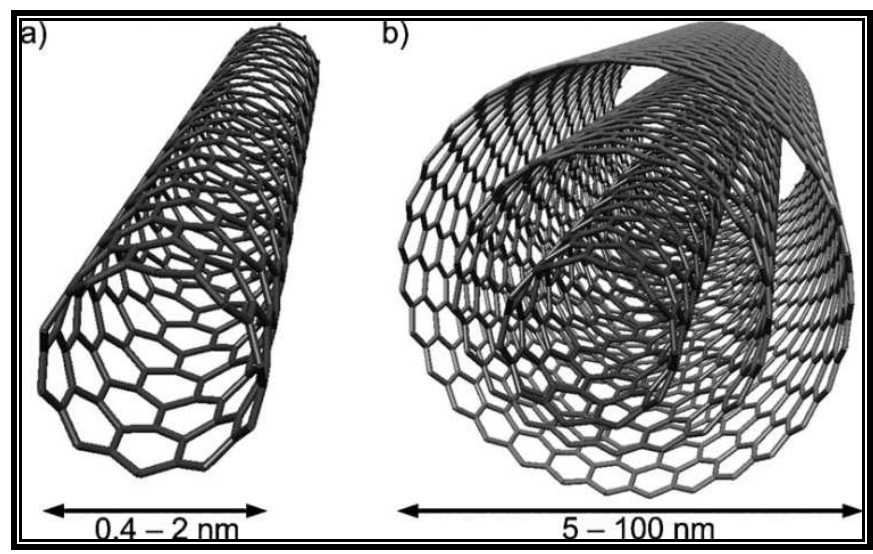

Fig 1. Two CNT variants: (a) a SWCNT and (b)a MWCNT

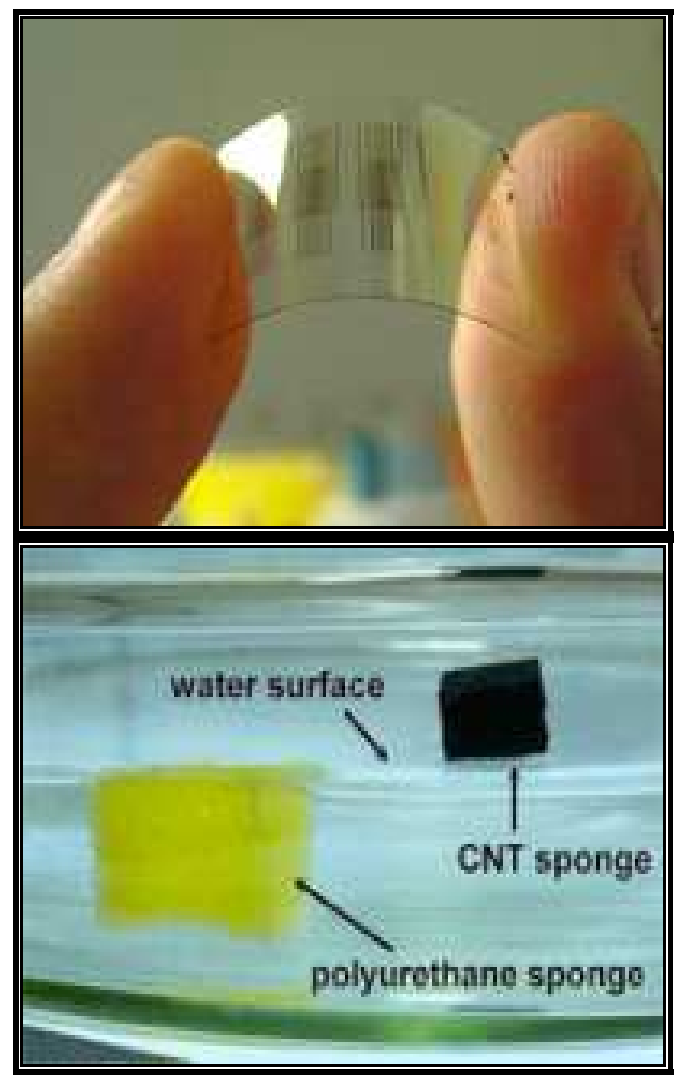

Fig 2. Energy storage devices of carbon nanotubes

\section{Electronics and Microchip Manufacturing}

The fact that nanotubes are very good conductors, and that they also appear as semiconductors or even insulators, makes them very useful for minuscule electronic devices like logic circuits composed of several transistors. The 
making of tiny circuits might be promising for the semiconductor industry. This industry is focused to make computer chips smaller every year, but also has on making them as cheap as possible. The conventional method of photolithography uses masks which have to be patterned once, which is time consuming and expensive, but they can be used many times. To create a normal chip, several masks are required. In comparison to the making of the mask, the manufacturing of the chips themselves is very rapid. Therefore, only large production quantities are profitable. The problem is that, this technique is reaching the limit of the smallest possible width of the lines (about $150 \mathrm{~nm}$ ). Smaller wavelengths have to be used to prevent blurring, which occurs when the features get too small. A microchip of carbon nanotubes is shown in Fig.3.

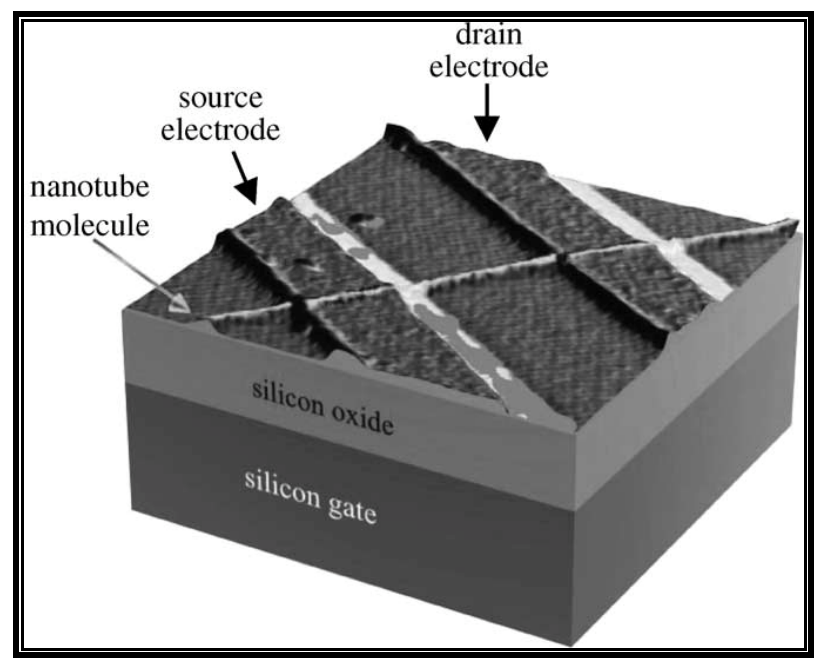

Fig 3. Microchip of carbon nanotubes

\section{Biomedicine and Nanotechnology}

Biosensors are simple, inexpensive measurement systems that use biological molecules usually enzymes, antibodies, or nucleic acids - to recognize simple molecules of interest via hydrogen bonding, charge-charge interactions and other biochemical interactions, and to provide molecular information. Recent advances in the field of nanotechnology and processing have resulted in solid-state biosensors offering unprecedented capabilities for genetic screening and detection. Research is currently in progress to fabricate nanomaterials by integrating nanofabrication and chemical functionalization, particularly in the case of nanoelectrode assemblies interfaced with biomolecules for the development of biosensors. These sensors will be integrated into the next-generation 'gene-chips', especially where the detection of less than an attomolecule is critical. The recent discovery of quantum confined particles or quantum dots (QDs) having unique optical and electronic properties, such as the size and composition-tunable fluorescence emission from visible to infrared wavelengths, a large absorption coefficient across a wide spectral range and a very high level of brightness and photo-stability, will lead to the development of multifunctional nanoparticle probes for cancer targeting and real-time in-vivo imaging in living cells.

Open-ended nanostraws could penetrate into a cellular structure for chemical probing or could be used as ultra small pipettes to inject molecules into living cells. Using computer simulations, it has been shown [13] that water molecules will quickly enter and flow through a carbon nanotube of $8 \mathrm{~nm}$ in diameter. A separate set of simulations shows that certain organic molecules can also flow through such nanotubes. The nanotubes conduct water at a rate similar to that of certain channels in the kidneys. These unusual transport properties of carbon nanotubes might be used in biomedical applications, such as highly targeted drug delivery.

\section{Automotive and Aerospace Industry}

Carbon fibre is already used to strengthen a wide range of materials, and the special properties of carbon nanotubes mean that they could be the ultimate high-strength fibre, which has a tensile strength 20 times, and a strength-to weight ratio100 times that of steel. Nanotubes have already been used for the reinforcement of nanostructural composite materials, polymers and concrete. Being stronger than diamond, it is the strongest material known. Because of their low density, these materials are very useful for cars, aeroplanes and space vehicles [14]. A carbon nanotube rope using an artificial impression of a space elevator is shown in Fig.4.

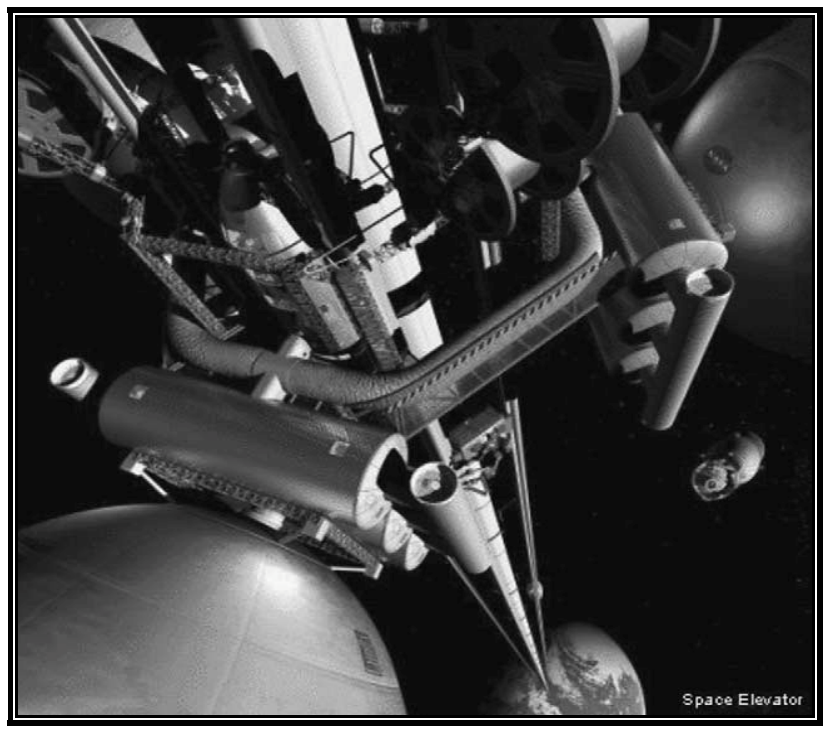

Fig 4. Artificial impression of a space elevator using a carbon nanotube rope

\section{Conclusion}

Carbon nanotubes have been the subject of discussion in recent years due to their diversified structures, properties and variety of applications. Nanotubes are commonly found 
in laboratories today, and research is stimulated by the large amounts of money invested in it. Some companies are already specializing in the production of carbon nanotubes, and giving research a boost. It is not known exactly when and where nanotubes will find their applications on a massive scale, but it seems highly likely. That is the trigger that makes the research on them so exciting.

\section{Acknowledgement}

The authors thank the Management of Sree Sastha Institute of Engineering and Technology, Chembarambakkam, Chennai -600123 for their encouragements throughout this work.

\section{References}

[1] S.I. Cha, K.T. Kim, K.H. Lee, C.B. Mo, Y.J. Jeong, S.H. Hong. Carbon 46 (2008) 482.

[2] H.J. Lee, S.W. Han, Y.D. Kwon, L.S. Tan, J.B. Baek. Carbon $46(2008) 1850$.

[3] W. Lu, N. Li, W. Chen, Y.Yao. Carbon 47 (2009) 3337.

[4] L. Wang, D. Zhu, L. Duan, W. Chen. Carbon 48 (2010) 3906.
[5] L. Rotkina, J. F. Lin, J.P. Bird, Appl. Phys. Lett. 83, 4426 (2003).

[6] S. J. Tans, R. M. Verschueren, C. Dekker, Nature 393, 49 (1998).

[7] Ch. Emmenegger, Ph. Mauron, P. Sudan, P.Wenger, V. Hermann, R. Gallay, A. Zuttel, J. Power Source 124, 321 (2003).

[8] M.S. Fuhrer, B.M. Kim, T. Durkop, T. Brintlinger, Nano Lett. 2, 755 (2002).

[9] N. S. Lee, D.S. Chung, I.T. Han, J.H. Kang, Y.S. Choi, H.Y. Kim, S.H. Park, Y. W. Jin,W. K. Yi, M. J. Yun, J. E. Jung, C. J. Lee, J. H. You, S. H. Jo, C. G. Lee, J. M. Kim, Diamond and Related Materials 10, 265 (2001).

[10] W.B. Choi, private communication, (2001).

[11] Y. Saito, T. Nishiyama, T. Kato, S. Kondo, T. Tanaka, J. Yotani, S. Uemura, Mol. Cryst.Liquid Cryst 387, 303 (2002).

[12] J. Yoon, C.Q. Ru, A. Mioduchowski, Proceedings IEEE ICMENS, (2003).

[13] Hummer, et al. Carbon nanotubes turn on water flow. Technology review; (2001) 29.

[14] Mamalis AG. New trends in nanotechnology. In: Proceedings of the Symposium on the 50th Anniversary of Department of Manufacturing Engineering of Budapest University of Technology and Economics, Budapest, Hungary; 2001. 